\title{
Intrahepatic Cholangiocarcinoma by AJCC v7 Stage
}

National Cancer Institute

\section{Source}

National Cancer Institute. Intrahepatic Cholangiocarcinoma by A/CC v7 Stage. NCI

Thesaurus. Code C134514.

A term that refers to the staging of intrahepatic cholangiocarcinoma according to the American Joint Committee on Cancer, 7th edition. 